\title{
Volvulus of the Wandering Spleen
}

\author{
Kalaventhan $\mathrm{P}^{1 *}$, Sarangan $\mathrm{S}^{2}$ and Sutharshan $\mathrm{V}^{3}$ \\ ${ }^{1}$ Department of Orthopaedics, Ministry of Health, Thandavanvely, Batticaloa, Srilanka \\ ${ }^{2}$ Faculty of Medicine, Department of Surgery, University of Colombo, Sri Lanka \\ ${ }^{3}$ Department of Surgery, Teaching Hospital Jaffna, Northern Province, Srilanka
}

*Corresponding author: Kalaventhan P, PG Trainee, Department of Orthopaedics, Ministry of Health, Thandavanvely, Batticaloa, Srilanka, Tel: +94 212 223348; E-mail: kalaventhansurgery@gmail.com

Rec Date: September 24, 2017, Acc Date: September 29, 2017, Pub Date: September 30, 2017

Citation: Kalaventhan P, Sarangan S, Sutharshan V (2017) Volvulus of the Wandering Spleen. Med Case Rep Vol.3 No.3:25.

\section{Abstract}

Wandering spleen is a rare condition characterized by the absence or underdevelopment of one or all of the ligaments that hold the spleen in its normal position in the left upper quadrant of the abdomen. Wandering spleen and gastric volvulus share a common cause, the absence of an intraperitoneal visceral ligament. Abnormal location of the spleen, which is called, wandering spleen, results from laxity or absence of the splenic pedicle. In the presence of an elongated splenic pedicle, torsion of the spleen or neighboring organs may occur, which results in acute or chronic abdominal pain. Here, we describe the case of a 10-year-old girl was admitted with right side upper abdominal pain of two days' duration.

Keywords: Volvulus; Spleen; Laparotomy; Splenopexy

\section{Introduction}

Wandering spleen is not a common condition characterized by the absence of ligamentous attachment to the left hypochondrium [1,2]. Here, we report a rare case of wandering spleen in association with situs inversus which is complicated by volvulus.

\section{Key Points}

The diagnosis and the viability of the wandering spleen can be made on the basis of the sonography and duplex study of the abdomen. But, volvulus deserved emergency laparotomy to perform splenopexy or splenectomy according to the viability of the spleen.

\section{Case Presentation}

A 10-year-old girl was admitted with right side upper abdominal pain of two days' duration. She also complained of vomiting in the incipient stages of abdominal pain and it settled on admission. Initial examination revealed that, she was afebrile, tachycardic and tachypnoeic. She had rigid abdomen with right hypochondrial tenderness.

Blood analysis was normal except increased white cell count and neutrophil leukocytosis. Ultrasound scan of the abdomen showed situs inversus and altered echogenicity of the spleen.

Laparotomy was performed and found to have situs inversus of the stomach, and intestines. The liver and the gallbladder were in the normal position. The spleen was found to be completely free floating without any ligamentous attachments and it was infarcted due to the volvulus (Figure 1). Pus was found in the pelvis. Splenectomy was done and the recovery was uneventful.

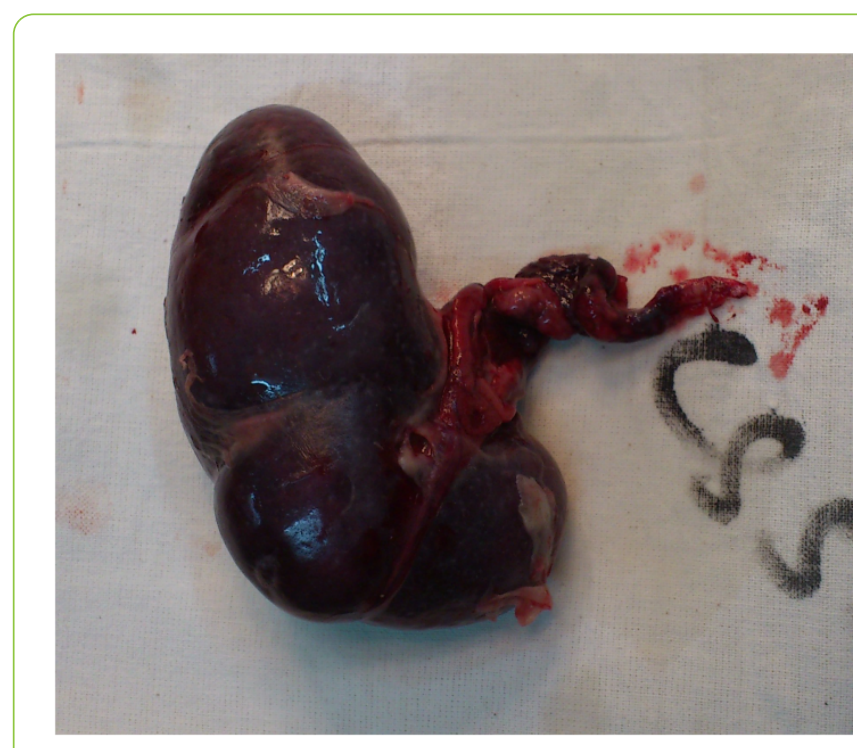

Figure 1 The infarcted spleen (Absence of the dorsal mesentry can be demonstrable in this specimen).

\section{Discussion}

The wandering spleen is only attached by an elongated vascular pedicle, leaving it to wander any part of the abdomen or pelvis [2]. The situs inversus is the shortened form of the Latin" situs inversus viscerum", is a term used to describe the 
inverted position of chest and abdominal organs [3]. Both are congenital malformations of peritoneal reflexions.

Most presentations are younger than 01 year; the male to female ratio is $6: 12$. In intrauterine life, the failed fusion between the dorsal mesogastrium and the posterior peritoneum, leading to absence of formation of supporting ligaments o spleen $[2,4,5]$. The mal-attached spleen has the increased risk of torsion and it can lead to splenic ischemia and infarction [2]. Fifteen percent of patients are asymptomatic, $55 \%$ are present with abdominal pain and $90 \%$ are presented with palpable mass in the abdomen $[2,5]$. Sixty four percent of paediatric patients complicated with torsion [2]. The splenic torsion is usually clockwise [2].

\section{Conclusion}

The abdominal sonography is the preliminary study to find out the absence or presence of the spleen in the left hypochondrium [1,2]. In addition, a duplex study can be conducted to assess the splenic blood flow [2]. Computed tomography (CT) determines the location of the spleen and the malposition of surrounding viscera such as situs inversus, gastric malposition [2]. In addition, infarction of the spleen and involvement of the pancreatic tail and surrounding fat also can be appreciated in CT [2].

Splenopexy is the surgical procedure to prevent torsion when a viable wandering spleen is found at surgery [2].

\section{References}

1. Salomonowitz E, Frick MP, Lund G (1984) Radiological diagnosis of wandering spleen complicated by splenic volvulus and infarction. Gastroint Radiol 9: 57-59.

2. Tzu H, Liu M, Lau KK (2007) Wandering spleen: An unusual association with gastric volvulus. Am J Gastroentol p. 188.

3. Complete situs inversus (2015) Available from: Radiopedia.org/ article/situs-inversus/

4. Erich W, Pollak, Henry $T$ (1977) Volvulus of the spleen. JAMA 237: 469-470.

5. Grumillier P, Cohen P, Fremont B (1997) Splenic volvulus on a mobile spleen. J Chir (Paris) 134: 444-446. 